\title{
On the use of the least common multiple to build a prime-generating recurrence
}

\author{
Serafín Ruiz-Cabello
}

April 21, 2022

\begin{abstract}
We study a recursively defined sequence which is constructed using the least common multiple. Several authors have conjectured that every term of that sequence is 1 or a prime. In this paper we show that this claim is connected to a strong version of Linnik's theorem, which is yet unproved. We also study a generalization that replaces the first term by any positive integer. Under this variation now some composite numbers may appear. We give a full characterization for these numbers.
\end{abstract}

\section{Introduction}

In 2003 Matthew Frank introduced the sequence $\left\{a_{n}^{\prime}\right\}$ defined by

$$
a_{n}^{\prime}=\left\{\begin{array}{ll}
7 & \text { for } n=1 \\
a_{n-1}^{\prime}+\operatorname{gcd}\left(n, a_{n-1}^{\prime}\right) & \text { for } n \geq 2
\end{array} .\right.
$$

Computations suggested that the difference between consecutive terms, $b_{n}^{\prime}:=a_{n}^{\prime}-a_{n-1}^{\prime}$ for $n \geq 2$, was always 1 or a prime. This result was proved by Rowland Row08. Chamizo, Raboso, and the current author proved that the sequence $\left\{b_{n}^{\prime}\right\}$ contained infinitely many primes [CRR11] and several interesting variants of this sequence were studied [Clo11. In 2008 Benoit Cloitre considered

$$
a_{n}=\left\{\begin{array}{ll}
1 & \text { for } n=1 \\
a_{n-1}+\operatorname{lcm}\left(n, a_{n-1}\right) & \text { for } n \geq 2,
\end{array} \quad \text { and } \quad b_{n}=\frac{a_{n}}{a_{n-1}}-1, n \geq 2 .\right.
$$

It is easy to check that every term of $\left\{a_{n}\right\}$ is nonzero and a multiple of the previous one. Thus the sequence $\left\{b_{n}\right\}$ is well defined and all terms are positive integers. Let us take a look at the first ones: 
$\left\{b_{n}\right\}=\{2,1,2,5,1,7,1,1,5,11,1,13,1,5,1,17,1,19,1,1,11,23,1,5,13,1,1,29,1$, $31,1,11,17,1,1,37,1,13,1,41,1,43,1,1,23,47,1,1,1,17,13,53,1,1,1,1,29,59,1$, $61,1,1,1,13,1,67,1,23,1,71,1,73,1,1,1,1,13,79,1,1,41,83,1,1,43,29,1,89,1,13$, $23,1,47,1,1,97,1,1,1,101,1,103,1,1,53,107,1,109,1,1,1,113,1,23,29,1,59,1,1,1$, $61,41,1,1,1,127,1,43,1,131,1,1,67,1,1,137,1,139,1,47,71,1,1,29,73,1,1,149,1$, $151,1,1,1,1,1,157,1,53,1,1,1,163,1,1,83,167,1,13,1,1,43,173,1,1,1,59,89,179$, $1,181,1,61,1,1,1,1,47,1,1,191,1,193,1,1,1,197,1,199,1,67,101, \ldots\}$

At first glance we notice two details. It seems that the sequence does not contain any composite number and hence there are just ones and primes. Moreover, it looks like every prime number appears, except maybe 3 . In consequence, one may conjecture these two facts:

Conjecture 1. For any $n \geq 2, b_{n}$ is either 1 or a prime number.

Conjecture 2. The sequence $\left\{b_{n}\right\}$ contains every prime number other than 3 , which never appears.

As far as we know, there is no proof for Conjecture 1, although numerical evidences suggest that it probably holds. On the other hand, M. Schepke proved [Sch, Thm. 3.10] that for any prime distinct than 3 , the term $b_{p}$ is equal to $p$. Hence, the remaining open question for Conjecture 2 is if number 3 can ever show up in the sequence.

In Section 2 we give a full proof for Conjecture 2 (direct consequence of Propositions 5 and 6) and a sufficient condition (see Proposition 4) for Conjecture 1 that links it to a well-known theorem proved by Linnik. In a general way, Linnik's theorem asserts that there exist positive constants $c$ and $L$ such that the first prime in the arithmetic progression $a, a+d, a+2 d, \ldots$ is less than $c d^{L}$ for any coprime integers $a$ and $d$ with $1 \leq a<d$ [Lin44], for some positive constants $c$ and $L$. The best known bound for $L$ is 5 [Xyl11], and it is conjectured that the theorem is still true for $L=2$ and $c=1$ [Hea92]. This stronger statement implies that for any prime $p$, the sequence $p-1,2 p-1, \ldots p^{2}-1$ should contain at least one prime number. If this claim turns out to be false even for a single prime, then Conjecture 1 would be false too (as a consequence of Proposition 4).

In Section 3 we deal with (1) when $a_{1}$ is not 1 but any positive integer. Under this variant, now it is possible to find composite odd numbers on the sequence, depending on the value of $a_{1}$. We shall give a necessary and sufficient condition for such numbers to appear or not on these sequences (see Theorem 13). Finally, Section 4 contains some examples and calculations that support our claims and explain the behavior of the sequence (1). 


\section{Auxiliary tools and proofs of the main results}

As we shall prove, the key tool to explain why Conjecture 1 seems to hold is the fact that for any prime $p$ and every integer $k \geq 1$, computations suggest that there are at least $k$ primes which are congruent to -1 modulo $p$ and less than $p^{k+1}$ (see Section 4 for further information). And actually this is more than enough. Here and subsequently, $\pi(x ; q, a)$ denotes the number of primes which are less than $x$ and congruent to $a$ modulo $q$, for integers $1 \leq a \leq q$.

Conjecture 3. For any prime $p$ and any integer $k \geq 1$,

$$
\pi\left(p^{k+1} ; p, p-1\right) \geq k
$$

For $k=1$, there are some cases on which the equality in (2) holds. Namely, 2, 5 and 13 seem to be the only ones. For larger values of $p$, the quantity $\pi\left(p^{k+1} ; p, p-1\right)$ grows quickly as suggested by Montgomery's conjecture [MV07, §13]. Apparently, (2) is always a strict inequality for $k>1$ (see Figure 1 and Table 6 on page 13). It is not possible however to claim that no counterexamples can ever be found.

Proposition 4. If Conjecture 3 holds, then $b_{n}$ can only be 1 or the largest prime factor of $n$ for every $n \geq 2$. Hence, Conjecture 3 implies Conjecture 1.

Proposition 5. For any prime $p \neq 3$, we have $b_{p}=p$.

Proposition 6. Any positive integer $n$ satisfies $b_{n} \neq 3$.

To prove these three Propositions, we shall employ the following Lemma:

Lemma 7. Sequences $\left\{a_{n}\right\}$ and $\left\{b_{n}\right\}$ satisfy the following results:

1. $a_{n-1}$ is greater than $n$ for every $n \geq 4$.

2. If a prime $p$ divides $a_{n}$ for a given $n \geq 1$, then $p \leq n+1$.

3. Every term in $\left\{b_{n}\right\}$ can be written as

$$
b_{n}=\frac{1}{a_{n-1}}\left(a_{n}-a_{n-1}\right)=\frac{\operatorname{lcm}\left(n, a_{n-1}\right)}{a_{n-1}}=\frac{n}{\operatorname{gcd}\left(n, a_{n-1}\right)} .
$$

In particular, $b_{n}$ is always a divisor of $n$.

Proof: The first point follows from the fact that every term of $\left\{a_{n}\right\}$ is at least twice as big as the previous one, and from $a_{3}>4$. For the second one, it is enough to note $a_{n}=$ $a_{n-1}\left(1+\operatorname{lcm}\left(n, a_{n-1}\right) / a_{n-1}\right) \leq a_{n-1}(1+n)$ and to apply induction. Finally, the last one is obtained from (1) using $a b=\operatorname{gcd}(a, b) \cdot \operatorname{lcm}(a, b)$. 
Proof of Proposition 5. The case $p=2$ is straightforward. For $p \geq 5$ prime we write $b_{p}=$ $p / \operatorname{gcd}\left(p, a_{p-1}\right)$ from (3). It is sufficient to prove that $p$ and $a_{p-1}$ are coprime. Clearly $p$ does not divide $a_{p-2}$ (as a consequence of the second point of Lemma 7); on the other hand, since $p-1$ is even, 2 is a common divisor of it with $a_{p-1}$, because $a_{n}$ is even for $n \geq 3$. Hence

$$
a_{p-1}=a_{p-2}+\operatorname{lcm}\left(p-1, a_{p-2}\right) \leq a_{p-2}+\frac{p-1}{2} a_{p-2},
$$

and then $a_{p-1} / a_{p-2} \leq(p+1) / 2<p$. As $p$ does not divide the product $\left(a_{p-1} / a_{p-2}\right) a_{p-2}$ and is a prime, we conclude that it is coprime with $a_{p-1}$, which completes the proof.

Proof of Proposition 4. Conjecture 1 holds for $b_{2}$ and $b_{3}$. Let us fix $m \geq 4$ (by Lemma 7, this implies $m<a_{m-1}$ ), and let $p$ be the largest prime factor of $m$. We only need to show that $m / p$ is a divisor of $a_{m-1}$, since in that case $\operatorname{lcm}\left(m, a_{m-1}\right)$ can only be $a_{m-1}$ or $p a_{m-1}$ and thus $b_{m}$ can only be 1 or $p$ by (3).

The crucial fact is that every term in the sequence $\left(a_{n}\right)$ is a multiple of the preceding one (in fact, of $a_{n}^{\prime}$ for every $\left.n^{\prime}<n\right)$. From the first equality in (3), it is clear that $a_{n}=a_{n-1}\left(b_{n}+1\right)$, and in consequence every $a_{n}$ can be expressed in terms of the sequence $\left(b_{n}\right)$, since by induction we have

$$
a_{n}=\prod_{k=2}^{n}\left(1+b_{k}\right), \quad n \geq 2 .
$$

For a fixed integer $m$, we consider its unique prime factorization,

$$
m=p_{1}^{\alpha_{1}} p_{2}^{\alpha_{2}} \cdot \ldots \cdot p_{k}^{\alpha_{k}}
$$

with $p=p_{k}$ and (only if $k>1$ ) $p_{1}, \ldots, p_{k-1}<p$. For $j<k$, we have $m \geq p_{j}^{\alpha_{j}} \cdot p>p_{j}^{\alpha_{j}+1}$. By (2), there exist at least $\alpha_{j}$ primes $q_{1}, q_{2}, \ldots, q_{\alpha_{j}}$ less than $p_{j}^{\alpha_{j+1}}$ (and hence less than $m$ ) which are congruent with -1 modulo $p_{j}$. For each one of them, Proposition 5 implies $b_{q_{l}}=q_{l}$ and therefore $a_{q_{l}}=a_{q_{l}-1}\left(1+q_{l}\right)$. Since $p_{j}$ divides $1+q_{l}$, it must divide $a_{q_{l}}$ at least one more time than it divides $a_{q_{l}-1}$. Thus $a_{m-1}$ contains the factor $p_{j}$ at least $\alpha_{j}$ times.

The case $p_{k}$ is very similar, except for the fact that now we cannot guarantee $m \geq p_{k}^{\alpha_{k}+1}$ but only $m \geq p_{k}^{\alpha_{k}}$. We apply the same argument as above in order to prove that $p_{k}$ divides $a_{m-1}$ at least $\alpha_{k}-1$ times and the proof is finished.

For the Proof of Proposition 6 we also need an explicit lower bound on the number of prime numbers which are congruent to 2 modulo 3. Namely we use Dus02]

$$
\pi(x ; 3,2)>\frac{x}{2 \log x} \quad \text { for } \quad x \geq 151 .
$$


Lemma 8. For every integer $k \geq 0$, we have

$$
\pi\left(3^{k} ; 3,2\right) \geq k .
$$

Proof: It is easy to check that (6) holds for $0 \leq k \leq 4$. For larger values of $k$ we use (5) and the fact that $3^{x} x^{-2}$ is an increasing function from $x=5$ onwards:

$$
\pi\left(3^{k} ; 3,2\right)>\frac{3^{k}}{(\log 9) k} \geq \frac{3^{5}}{(\log 9) 5^{2}} k>k .
$$

Note that Lemma 8 actually is a stronger version of (2) for $p=3$. This is the only prime such that $p-1$ is also a prime, and that is the reason why 3 does not appear in $\left\{b_{n}\right\}$.

Proof of Proposition 6: By (3), it is sufficient to show that $3^{k}$ divides $a_{3^{k}}$ for any positive integer $k$. Lemma 8 implies that there exist primes $p_{1}, p_{2}, \ldots p_{k}$ less than $3^{k}$ which are congruent to -1 modulo 3. By Lemma 5, $b_{p_{j}}=p_{j}$, and hence $a_{p_{j}}=a_{p_{j-1}}\left(p_{j}+1\right)$ is a multiple of 3 . The proof is concluded using that every $a_{m}$ is a multiple of $a_{m-1}$ and applying induction.

\section{Generalization}

Now a natural question arises: What happens if the initial condition in (1) is not 1 but any possitive integer? For a fixed $s \geq 1$ we define

$$
a_{n}^{s}=\left\{\begin{array}{ll}
s & \text { for } n=1 \\
a_{n-1}^{s}+\operatorname{lcm}\left(n, a_{n-1}^{s}\right) & \text { for } n \geq 2,
\end{array} \quad \text { and } \quad b_{n}^{s}=\frac{a_{n}^{s}}{a_{n-1}^{s}}-1, n \geq 2 .\right.
$$

The relation between differents sequences of this family is surprising (see Table 1). The first thing we notice is that they all seem to be very similar, with little variations that may depend on common divisors of given values of $n$ and $s$. Also, it is clear that Conjecture 1 cannot be generalized for every $s$. We can see on Table 1 that for instance the sequence $\left\{b_{n}^{10}\right\}$ contains a composite number, a 9. For suitable values of $s$ (that, as we shall discuss later, strongly depend on the composite number $m$ we want to find), many more counterexamples are found. For instance,

$$
b_{21}^{24310}=21, \quad b_{25}^{19}=25, \quad b_{27}^{43010}=27, \quad b_{35}^{7163}=35 .
$$

See Tables 2 and 3 from page 11 onwards for more examples. There are also many numbers that never appear. It is not hard to prove (see Proposition 12) that no even number greater than 2 can be found in any sequence. A little more is required to show that, for instance, $b_{n}^{s}$ is not 15 for any pair $(n, s)$. According to this, from now on we shall classify composite numbers into two different groups: 


\begin{tabular}{|l||l|l|l|l|l|l|l|l|l|l|l|l|l|l|l|l|}
\hline$a_{1}^{s}$ & $b_{2}^{s}$ & $b_{3}^{s}$ & $b_{4}^{s}$ & $b_{5}^{s}$ & $b_{6}^{s}$ & $b_{7}^{s}$ & $b_{8}^{s}$ & $b_{9}^{s}$ & $b_{10}^{s}$ & $b_{11}^{s}$ & $b_{12}^{s}$ & $b_{13}^{s}$ & $b_{14}^{s}$ & $b_{15}^{s}$ & $b_{16}^{s}$ & $b_{17}^{s}$ \\
\hline 1 & 2 & 1 & 2 & 5 & 1 & 7 & 1 & 1 & 5 & 11 & 1 & 13 & 1 & 5 & 1 & 17 \\
\hline 2 & 1 & 3 & 1 & 5 & 1 & 7 & 1 & 3 & 5 & 11 & 1 & 13 & 1 & 5 & 1 & 17 \\
\hline 3 & 2 & 1 & 2 & 5 & 1 & 7 & 1 & 1 & 5 & 11 & 1 & 13 & 1 & 5 & 1 & 17 \\
\hline 4 & 1 & 3 & 1 & 5 & 1 & 7 & 1 & 3 & 5 & 11 & 1 & 13 & 1 & 5 & 1 & 17 \\
\hline 5 & 2 & 1 & 2 & 1 & 1 & 7 & 1 & 1 & 1 & 11 & 1 & 13 & 1 & 1 & 1 & 17 \\
\hline 6 & 1 & 1 & 1 & 5 & 1 & 7 & 1 & 1 & 5 & 11 & 1 & 13 & 1 & 5 & 1 & 17 \\
\hline 7 & 2 & 1 & 2 & 5 & 1 & 1 & 1 & 1 & 5 & 11 & 1 & 13 & 1 & 5 & 1 & 17 \\
\hline 8 & 1 & 3 & 1 & 5 & 1 & 7 & 1 & 3 & 5 & 11 & 1 & 13 & 1 & 5 & 1 & 17 \\
\hline 9 & 2 & 1 & 2 & 5 & 1 & 7 & 1 & 1 & 5 & 11 & 1 & 13 & 1 & 5 & 1 & 17 \\
\hline 10 & 1 & 3 & 1 & 1 & 3 & 7 & 1 & 9 & 1 & 11 & 1 & 13 & 1 & 1 & 1 & 17 \\
\hline 11 & 2 & 1 & 2 & 5 & 1 & 7 & 1 & 1 & 5 & 1 & 1 & 13 & 1 & 5 & 1 & 17 \\
\hline
\end{tabular}

Table 1: First values of $\left\{b_{n}^{s}\right\}$ for $1 \leq s \leq 11$.

Definition 9. Given a composite integer $m \geq 3$, we call it a present number if there exist $n$ and $s$ such that $b_{n}^{s}=m$. Otherwise, we call it an absent number.

So, besides the first open question of this chapter (can Conjectures 1 and 2 be generalized for a fixed $s$ ?), another one -which turns out to be much more interesting- arises: can we classify composite numbers into absent ones and present ones? And the answer is positive; Theorem 13 (among with Propositions 11 and 12) gives a full characterization.

But first let us say what we can now about the presence of composite numbers on the sequence $\left\{b_{n}^{s}\right\}_{n \geq 1}$ for a given $s$. Depending on the factors of $s$, sometimes it is possible to establish an algorithm in order to show that $s$ is allowing any present number to appear. The simplest examples are $s=19$ and $s=103$ (or many of their multiples), that lead to $b_{5^{2}}^{19}=5^{2}$ and $b_{13^{2}}^{103}=13^{2}$. As we discussed before, this is related to the fact that 5 and 13 are probably the only odd primes such that the equality in (2) holds for $k=1$. If we get a negative result for that particular $s$, then Conjecture 1 can be generalized to $\left\{b_{n}^{s}\right\}$, by using a slightly modified version of (2) (that changes for every $s$ ). It seems difficult, however, to establish a general constructive method for any large $s$. Regarding Proposition 5 , it can be extended for any $s$ (see Proposition 11), but not Proposition 6 (see the remark after Proposition 12). First, we need to introduce this extended version of Lemma 7 .

Lemma 10. Fix $s \geq 1$. Sequences $\left\{a_{n}^{s}\right\}$ and $\left\{b_{n}^{s}\right\}$ satisfy the following results:

1. $a_{n-1}^{s}$ is greater than $n$ for every $n \geq 4$ and $s \geq 1$.

2. If a prime $p$ divides $a_{n}^{s}$ for a given $n \geq 1$ and $p \nmid s$, then $p \leq n+1$. 
3. $b_{n}^{s}$ can be written as

$$
b_{n}^{s}=\frac{\operatorname{lcm}\left(n, a_{n-1}^{s}\right)}{a_{n-1}^{s}}=\frac{n}{\operatorname{gcd}\left(n, a_{n-1}^{s}\right)} .
$$

In particular, $b_{n}^{s}$ is always a divisor of $n$.

4. A given $a_{n}^{s}$ for $n \geq 2$ can be expressed in terms of $b_{2}^{s}, b_{3}^{s}, \ldots, b_{n}^{s}$. Namely,

$$
a_{n}^{s}=\prod_{k=2}^{n}\left(1+b_{k}^{s}\right), \quad n \geq 2 .
$$

Proof. Taking into account that

$$
\left(a_{2}^{s}, a_{3}^{s}, a_{4}^{s}\right)= \begin{cases}(2 s, 4 s, 8 s) & \text { if } s \equiv 0 \quad(\bmod 6) \\ (2 s, 8 s, 16 s) & \text { if } s \equiv 2,4 \quad(\bmod 6) \\ (3 s, 6 s, 18 s) & \text { if } s \equiv 1 \quad(\bmod 2)\end{cases}
$$

and that if $p$ divides $a_{n}$, then either $p \leq n+1$ or $p$ divides $s$, the proof of Lemma 7 is still suitable for $\left\{a_{n}^{s}\right\}$ and $\left\{b_{n}^{s}\right\}$. Note that (9) is obtained as (4).

Proposition 11. Fix an integer $s \geq 1$ and a prime $p \neq 3$. We have $b_{4}^{s}=b_{2}^{s}$ and

$$
b_{p}^{s}=\left\{\begin{array}{ll}
p & \text { if } p \nmid s \\
1 & \text { if } p \mid s
\end{array}, \quad b_{3}^{s}=\left\{\begin{array}{lll}
3 & \text { if } s \equiv 2,4 \quad(\bmod 6) \\
1 & \text { if } s \not \equiv 2,4 & (\bmod 6) .
\end{array}\right.\right.
$$

Proposition 12. Given any initial condition $s, b_{n}^{s}$ is always odd for $n \geq 5$.

Note that Proposition 11 is a generalization of Proposition 5. However, it is not always possible to do the same with Proposition 6, because for a large prime $s$ we cannot prove $b_{n}^{s} \neq s$ for every $n$ as we did for $\left\{b_{n}^{1}\right\}$ on Lemma 8 (this would need an explicit lower bound for $\pi(x ; s, s-1)$, which do not always exists for large values of $s)$. Hence a full generalization of Conjecture 2 holds whenever $s$ is not a large prime. Note also that combining these two Propositions it is clear that, if $m \geq 4$ is an even integer, then $b_{n}^{s} \neq m$ for every possible values of $n$ and $s$.

Proof of Proposition 11: By (10), the Proposition is straightforward for $n=2,3,4$. For a prime $p \geq 5$, if $p$ divides $s$, then $a_{p-1}$ is a multiple of $p$ and by (8), $b_{p}=p / \operatorname{gcd}\left(a_{p-1}, p\right)=1$. And if $p \nmid s$, we can proceed as in the proof of Proposition 5, getting that $p$ and $a_{p-1}^{s}$ are coprime and hence $b_{p}^{s}=p$. 
Proof of Proposition 12: We employ an explicit lower bound on the number of primes which are less than a fixed $x$ Ros41,

$$
\pi(x ; 2,1)>\frac{x}{\log x+2}-1 \quad \text { for } \quad x \geq 55 .
$$

Using this inequality and proceeding as in the proof of Lemma 8, it is easy to check that $\pi\left(2^{k}, 2,1\right) \geq k$ for $k \geq 3$. Combining this with (10), we have $8 \mid a_{7}^{s}$ and by induction, $2^{k} \mid a_{2^{k}-1}^{s}$ for $k \geq 3$.

For the next results, we shall use the following notation: for any two integers $q$ and $m$, we will write $q \triangleleft m$ if every prime divisor of $q$ is also a prime divisor of $m$ and $q<m$. For instance, $9 \triangleleft 15,27 \Varangle 15$ or $6 \Varangle 9$.

Theorem 13. Let $m \geq 3$ be an odd composite integer. It there exist $p$ and $q$ such that $p$ is a prime divisor of $m, q \triangleleft m$ and $q \equiv-1(\bmod p)$, then $m$ is an absent number. Otherwise there exists an integer $s$ such that $b_{m}^{s}=m$ and in consequence $m$ is a present number.

This result gives us a method in order to know whether an odd composite number is absent or not. It is clear from the Definition that every power of an odd prime is present. Actually, they are easy to find as explicit counterexamples. For instance,

$$
b_{7^{2}}^{533}=7^{2}, \quad b_{11^{2}}^{4687}=11^{2}, \quad b_{13^{2}}^{103}=13^{2}, \quad b_{5^{3}}^{s_{1}}=5^{3}, \quad \text { for } \quad s_{1}=19 \cdot 29 \cdot 59 \cdot 79 \cdot 89 \cdot 109 .
$$

For numbers with more than prime divisors, the condition of Theorem 13 is tricky and there are several possibilities, even with just two different primes. For instance, 21 is present, since $7 \not \equiv-1(\bmod 3)$. But 63 is absent, because $3^{3} \equiv-1(\bmod 7)$. The smallest absent number is 15 and, in the same way, every number of the form $3 p$ for a prime $p \equiv 5(\bmod 6)$ is an absent one. The more different divisors has $m$, the smallest are the chances for it to be present. The following result is straightforward:

Corollary 14. Every multiple of an absent number is also absent. Also, every divisor of a present number is present.

The proof of the theorem is split in two parts. For the one concerning the absent numbers, we use Lemma 15 and a recursive step (Proposition 16).

Lemma 15. If $m$ is an absent number for a given pair $(p, q)$, then we can always write $m=p q m_{0}$, and $p$ and $q$ are necessarily odd with $p<q$ and $\operatorname{gcd}(p, q)=1$.

Proposition 16. Let $m=p_{0}$ be an absent number for a pair $(p, q)$. For every integers $c, d \geq 1$ and $t:=p^{c} q^{d} m_{0}$, we claim that

1. If $b_{t}^{s}=1$, then $b_{p q t}^{s} \in\{1, p, q\}$. 
2. If $b_{t}^{s}=p$, then $b_{q t}^{s} \in\{1, p, q\}$.

3. If $b_{t}^{s}=q$, then $b_{p t}^{s} \in\{1, q\}$.

4. If $b_{t}^{s}=j \in\{1, p, q\}$, then $b_{k}^{s} \neq m$ for $t \leq k<(p q / j) t$.

Proof:

1. If $b_{t}^{s}=1$, then it means $p^{c} q^{d} \mid a_{t-1}^{s}$ by (8). We distinguish two cases now. If $q^{d+1} \mid a_{q t-1}^{s}$, then $p^{c} q^{d+1} \mid a_{p q t-1}^{s}$, and in consequence $b_{p q t}^{s}$ can only be 1 or $p$. If not, then we have $p^{c} q^{d} \mid a_{q t-1}^{s}$ and $q^{d+1} \nmid a_{q t-1}^{s}$, and hence $a_{q t}^{s}=(q+1) a_{q t-1}^{s}$, which implies $p^{c+1} q^{d} \mid a_{p q t-1}^{s}$. Thus $b_{p q t}^{s}$ can only be 1 or $q$.

2. If $b_{t}^{s}=p$, again by (8) we obtain $p^{c-1} q^{d} \mid a_{t-1}^{s}$ and $p^{c} \nmid a_{t-1}^{s}$. Since $p<q$, we have $t<p^{c-1} q^{d+1}=t q / p$. We distinguish two cases once more. If $q^{d+1}$ divides $a_{t q / p-1}^{s}$, then it divides $a_{q t-1}^{s}$ too. As a consequence, $b_{q t}^{s}$ can only be 1 or $p$. If $q^{d+1}$ does not divide $a_{t q / p-1}^{s}$ (and recalling that $\left.q^{d} \mid a_{t q / p-1}^{s}\right), b_{t q / p}^{s}$ is $q$ and $a_{t q / p}^{s}=(q+1) a_{t q / p-1}^{s}$. This implies $p \mid a_{t q / p}^{s}$ and $p \mid a_{p q t-1}^{s}$, from which $b_{p q t}^{s}$ can only be 1 or $q$.

3. The last case is $b_{t}^{s}=q$. As before, from it we obtain $p^{c} q^{d-1} \mid a_{t-1}^{s}$ and $q^{d} \nmid a_{t-1}^{s}$. But necessarily $a_{t}^{s}=(q+1) a_{t-1}^{s}$, and since $q \equiv-1(\bmod p)$, we conclude $p^{c+1} \mid a_{t}^{s}$ and $b_{p t}^{s}$ can only be 1 or $q$.

4. Finally, let us suppose $b_{t}^{s}=j \in\{1, p, q\}$. As every $b_{n}^{s}$ is a divisor of $n$, if we want to find $k$ such that $k \geq t$ and $b_{k}^{s}=m$, the least possible candidate is $t \cdot(p q / j)$. This proves the fourth and last point.

Proof of Theorem 13: We distinguish two cases. First, let us assume that for a particular $m$ there exist $p$ and $q$ as in the statement, that we can write in the form $p q m_{0}$ after Lemma 15 . Then it is a matter of combining the four properties of Proposition 16 and apply induction. For any $s \geq 1$, clearly $b_{p q}$ can only be $1, p$ or $q$ (if $q$ divides $a_{q-1}^{s}$, then $q \nmid b_{p q}^{s}$; and if not, then $a_{q}^{s}=(q+1) a_{q-1}^{s}$ and then $p \mid a_{p q-1}^{s}$ and $\left.p \nmid b_{p q}^{s}\right)$. We are now in one of the three possibilities, for $c=d=1$, and fall again in one of them, for $(c, d) \in\{(1,2),(2,2),(2,1)\}$. Iterating this process, we obtain an infinite sequence $\left\{\left(c_{j}, d_{j}\right)\right\}_{j \geq 1}$, with $c_{j} \leq c_{j+1} \leq c_{j}+1$ and $d_{j} \leq d_{j+1} \leq d_{j}+1$. And for every $n \geq 1$, there is a single $j$ such that $p^{c_{j}} q^{d_{j}} m_{0} \leq n<p^{c_{j+1}} q^{d_{j+1}} m_{0}$. By the fourth point, $b_{n}^{s} \neq m$.

The last case is when no pair $(p, q)$ satisfies the conditions of Theorem 13 for a fixed $m$. Then we take

$$
S=\{1 \leq l \leq m: \operatorname{gcd}(l, m)=1\}, \quad \text { and } \quad s:=\prod_{l \in S} l .
$$


Now let $p_{1}, \ldots, p_{k}$ be the prime divisors of number $m$. We employ the following sets:

$$
\begin{gathered}
P_{j}=\left\{1 \leq n \leq m: n \equiv-1 \quad\left(\bmod p_{j}\right)\right\}, \quad P=\bigcup_{j=1}^{k} P_{j}, \\
\widetilde{P}=\{1 \leq k p \leq m: k \triangleleft m, p \in P\}, \quad \text { and } \quad \widehat{P}:=\{1 \leq k p \leq m: k \in \mathbb{Z}, p \in P\} .
\end{gathered}
$$

Note that always $1 \notin P \subset \widetilde{P} \subset \widehat{P}$. Since clearly $m$ and $s$ are coprime, it is enough to prove $b_{n}^{s} \notin P$ for $2 \leq n<m$ because in that case, by $(9), m$ and $a_{m-1}^{s}$ are coprime too and then $b_{m}^{s}=m$ by (8). We shall prove this by complete induction. For the first step we recall that $s$ is an odd number, and hence $b_{2}^{s}=1 \notin P$ by Proposition 11. Now, for a given $2 \leq n<m$, let us suppose that $b_{j}^{s} \notin P$ for $j<n$, which implies that $n$ and $a_{n-1}^{s}$ are coprime. Using once more that $b_{n}^{s}$ is a divisor of $n$, we can restrict ourselves to the case $n \in \widehat{P}$; in other words, we can assume $n$ to be a multiple of an element of $P$. Now there are two possibilities. If $n$ is in $S$, then by (8), $b_{n}^{s}$ is 1 , which is not contained in $\widehat{P}$. Otherwise, we have $n \in \widehat{P} \backslash S$. Let us call $n_{0}$ the greatest multiple of $n$ such that $n_{0}$ and $m$ are coprime and write $n=n_{0} n_{1}$. Since $n \notin L$, this clearly implies $n_{0} \in L$ and $1<n_{1} \Varangle m$. Then

$$
b_{n}^{s}=\frac{n_{0} n_{1}}{\operatorname{gcd}\left(n_{0} n_{1}, a_{n-1}^{s}\right)}=n_{1} .
$$

Finally, every possible prime divisor of $n_{1}$ is contained in $\left\{p_{1}, \ldots, p_{k}\right\}$. But then there cannot exist $j$ such that $n_{1} \equiv-1\left(\bmod p_{j}\right)$, since $n_{1}$ is a divisor of a present number, and hence a present number too by Corollary 14 .

\section{Examples and tables}

As we have just seen, we know for sure that there are infinitely many values of $s$ such that $\left\{a_{n}^{s}\right\}$ contains composite numbers. However, very strong divisibility conditions are needed in order to find such numbers, that we called present (many composite numbers can never show up, as we stated on Theorem 13. So if we choose a random value for $s$, the odds say that probably that sequence is clean. Let us begin by taking a look at some examples of absent and present integers. Every power of a prime is present, the first ones are in Table 2. Note that, for any fixed present number $m$, the value $s$ that we get in the proof of the theorem is not necessarily the smallest possible. In the case $m=p^{k}$, actually it is enough to take the product of the primes contained in $P$, and that is what we do in our examples.

If $m$ has more than one prime divisor, then $m$ can be absent or present, as Definition 9 states. See Table 3. The first absent integers are 15, 33, 45, 51, 63, 65, 69, 75, 87 and 91. We stated that we can never find these integers in any generalized sequence. The rest of them are present, and by the proof of Theorem 13 , we can find suitable values of $s$ such that $b_{m}^{s}=m$. 


\begin{tabular}{|c|c|c|}
\hline$m$ & $p^{k}$ & $s$ \\
\hline 9 & $3^{2}$ & $2 \cdot 5$ \\
\hline 25 & $5^{2}$ & 19 \\
\hline 27 & $3^{3}$ & $2 \cdot 5 \cdot 11 \cdot 17 \cdot 23$ \\
\hline 49 & $7^{2}$ & $13 \cdot 41$ \\
\hline 81 & $3^{4}$ & $2 \cdot 5 \cdot 11 \cdot 17 \cdot 23 \cdot 29 \cdot 41 \cdot 47 \cdot 53 \cdot 59 \cdot 71$ \\
\hline 121 & $11^{2}$ & $43 \cdot 109$ \\
\hline 125 & $5^{3}$ & $19 \cdot 29 \cdot 59 \cdot 79 \cdot 89 \cdot 109$ \\
\hline 169 & $13^{2}$ & 103 \\
\hline 243 & $3^{4}$ & $\begin{array}{c}2 \cdot 5 \cdot 11 \cdot 17 \cdot 23 \cdot 29 \cdot 41 \cdot 47 \cdot 53 \cdot 59 \cdot 71 \cdot 83 \cdot 89 \cdot 101 \cdot 107 \cdot 113 \cdot 131 \cdot \\
\cdot 137 \cdot 149 \cdot 167 \cdot 173 \cdot 179 \cdot 191 \cdot 197 \cdot 227 \cdot 233 \cdot 239\end{array}$ \\
\hline 289 & $17^{2}$ & $67 \cdot 101 \cdot 271$ \\
\hline 343 & $7^{3}$ & $13 \cdot 41 \cdot 83 \cdot 97 \cdot 139 \cdot 167 \cdot 181 \cdot 223 \cdot 251 \cdot 293 \cdot 307$ \\
\hline 361 & $19^{2}$ & $37 \cdot 113 \cdot 151 \cdot 227$ \\
\hline 529 & $23^{2}$ & $137 \cdot 229 \cdot 367$ \\
\hline 625 & $5^{4}$ & $\begin{array}{c}19 \cdot 29 \cdot 59 \cdot 79 \cdot 89 \cdot 109 \cdot 139 \cdot 149 \cdot 179 \cdot 199 \cdot 229 \cdot 239 \cdot 269 \cdot 349 \cdot 359 \cdot \\
379 \cdot 389 \cdot 409 \cdot 419 \cdot 439 \cdot 449 \cdot 479 \cdot 499 \cdot 509 \cdot 569 \cdot 599 \cdot 619\end{array}$ \\
\hline 729 & $3^{5}$ & $\begin{array}{c}2 \cdot 5 \cdot 11 \cdot 17 \cdot 23 \cdot 29 \cdot 41 \cdot 47 \cdot 53 \cdot 59 \cdot 71 \cdot 83 \cdot 89 \cdot 101 \cdot 107 \cdot 113 \cdot 131 \cdot \\
\cdot 137 \cdot 149 \cdot 167 \cdot 173 \cdot 179 \cdot 191 \cdot 197 \cdot 227 \cdot 233 \cdot 239 \cdot 251 \cdot 257 \cdot 263 \\
\cdot 269 \cdot 281 \cdot 293 \cdot 311 \cdot 317 \cdot 347 \cdot 353 \cdot 359 \cdot 383 \cdot 389 \cdot 401 \cdot 419 \cdot 431 \\
\cdot 443 \cdot 449 \cdot 461 \cdot 467 \cdot 479 \cdot 491 \cdot 503 \cdot 509 \cdot 521 \cdot 557 \cdot 563 \cdot 569 \cdot 587 \\
\quad 593 \cdot 599 \cdot 617 \cdot 641 \cdot 647 \cdot 653 \cdot 659 \cdot 677 \cdot 683 \cdot 701 \cdot 719\end{array}$ \\
\hline 841 & $29^{2}$ & $173 \cdot 347 \cdot 463 \cdot 521 \cdot 811$ \\
\hline 961 & $31^{2}$ & $61 \cdot 433 \cdot 557 \cdot 619 \cdot 743 \cdot 929$ \\
\hline 1331 & $11^{3}$ & $\begin{array}{c}43 \cdot 109 \cdot 131 \cdot 197 \cdot 241 \cdot 263 \cdot 307 \cdot 373 \cdot 439 \cdot 461 \cdot 571 \cdot 593 \cdot 659 \cdot 769 \cdot \\
\cdot 857 \cdot 967 \cdot 1033 \cdot 1187 \cdot 1231 \cdot 1297 \cdot 1319\end{array}$ \\
\hline 1369 & $37^{2}$ & $73 \cdot 443 \cdot 739 \cdot 887 \cdot 1109$ \\
\hline r & $\vdots$ & \\
\hline
\end{tabular}

Table 2: Non trivial powers of odd primes, and the least $s$ such that $b_{m}^{s}=m$. 


\begin{tabular}{|c|c|c|c|c|}
\hline$m$ & $\prod p_{j}^{\alpha_{j}}$ & $\mathrm{G} / \mathrm{B}$ & $p, q$ & $s$ \\
\hline 15 & $3 \cdot 5$ & Good & $5 \equiv-1(3)$ & \\
\hline 21 & $3 \cdot 7$ & Bad & & $2 \cdot 5 \cdot 11 \cdot 13 \cdot 17$ \\
\hline 33 & $3 \cdot 11$ & Good & $11 \equiv-1(3)$ & \\
\hline 35 & $5 \cdot 7$ & Bad & & $13 \cdot 19 \cdot 29$ \\
\hline 39 & $3 \cdot 13$ & $\mathrm{Bad}$ & & $2 \cdot 5 \cdot 11 \cdot 17 \cdot 23 \cdot 29$ \\
\hline 45 & $3^{2} \cdot 5$ & Good & $\begin{array}{c}5 \equiv-1(3) \\
3^{2} \equiv-1(5)\end{array}$ & \\
\hline 51 & $3 \cdot 17$ & Good & $17 \equiv-1(3)$ & \\
\hline 55 & $5 \cdot 11$ & Bad & & $19 \cdot 29 \cdot 43$ \\
\hline 57 & $3 \cdot 19$ & $\mathrm{Bad}$ & & $2 \cdot 5 \cdot 11 \cdot 17 \cdot 23 \cdot 29 \cdot 37 \cdot 41 \cdot 47 \cdot 53$ \\
\hline 63 & $3^{2} \cdot 7$ & Good & $3^{3} \equiv-1(7)$ & \\
\hline 65 & $5 \cdot 13$ & Good & $5^{2} \equiv-1(13)$ & \\
\hline 69 & $3 \cdot 23$ & Good & $23 \equiv-1(3)$ & \\
\hline 75 & $3 \cdot 5^{2}$ & Good & $\begin{aligned} 5 & \equiv-1(3) \\
3^{2} & \equiv-1(5)\end{aligned}$ & \\
\hline 77 & $7 \cdot 11$ & Bad & & $13 \cdot 41 \cdot 43$ \\
\hline 85 & $5 \cdot 17$ & Bad & & $19 \cdot 29 \cdot 59 \cdot 67 \cdot 79$ \\
\hline 87 & $3 \cdot 29$ & Good & $29 \equiv-1(3)$ & \\
\hline 91 & $7 \cdot 13$ & Good & $13 \equiv-1(7)$ & \\
\hline 93 & $3 \cdot 31$ & Bad & & $\begin{array}{c}2 \cdot 5 \cdot 11 \cdot 17 \cdot 23 \cdot 29 \cdot 41 \cdot 47 \cdot 53 \cdot \\
\cdot 59 \cdot 61 \cdot 71 \cdot 83 \cdot 89\end{array}$ \\
\hline 95 & $5 \cdot 19$ & Good & $19 \equiv-1(5)$ & \\
\hline 99 & $3^{2} \cdot 11$ & Good & $11 \equiv-1(3)$ & \\
\hline 105 & $3 \cdot 5 \cdot 7$ & Good & $\begin{array}{c}3^{2} \equiv-1(5) \\
5 \cdot 7 \equiv-1(3) \\
7^{2} \equiv-1(5)\end{array}$ & \\
\hline 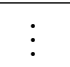 & & & & \\
\hline
\end{tabular}

Table 3: First odd integers with at least two different prime divisors. If they are present, the least $s$ such that $b_{m}^{s}=s$. If they are absent, all the counterexample pairs $(p, q)$. 


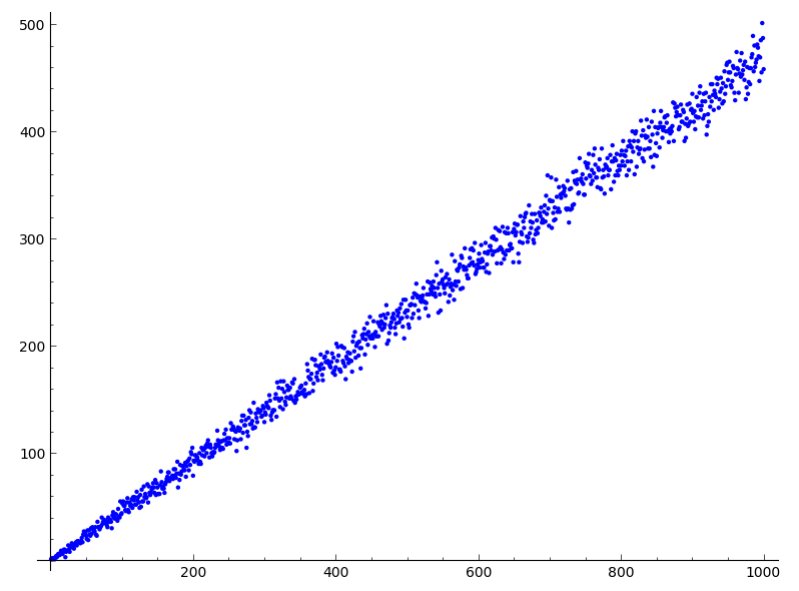

Figure 1: The first 1000 primes $p_{n}$ vs. $\pi\left(p_{n}^{2} ; p_{n}, p_{n}-1\right)$, which is never 0 in this range.

Again, it is not necessary to take so many factors to get $s$, but taking just the primes in $P$ is not enough. For instance, taking $s=3 \cdot 13 \cdot 19$, we need $43 \mid s$ to get $b_{m}^{s}=m$, although 43 is not congruent to -1 modulo 3,13 or 19 (the reason for this is the fact that $3 \cdot 43$ is congruent to -1 modulo 13$)$.

For larger values of $m$, usually more numbers are expected to be absent, as having more different factors creates more chances. However, picking products of two different large primes, we can always find arbitrarily large present numbers. It is not easy to describe all the pairs $(n, s)$ such that a present number $m$ satisfies $b_{n}^{s}=m$; if $n$ is greater than $m$, there are endless possibilities adding and removing factors from $s$.

On the other hand, given a specific value of $s$, is there a way to check if the sequence $\left\{b_{n}^{s}\right\}$ contains present numbers? Again, there is not a clean answer. As we discussed before, the number of times that a prime $p$ divides $a_{n}^{s}$ depends not only on the primes $q$ less than $n$ and such that $q \equiv-1(\bmod p)$, but on the factors $b_{j}^{s}$ with $j \leq n$ and $b_{j}^{s} \equiv-1(\bmod p)$ (and also on the factors of $s$ itself). If $s$ is not a huge number and we can manually check $O(s)$ steps in the sequence $b_{n}^{s}$, then are able to say if there are present numbers, or if we can establish the original conjecture for this particular value of $s$.

Finally, let us go back to the original sequence, (1), and say a few words about Conjecture 1. As we can see on Table 4, $\left\{a_{n}\right\}$ grows very fast. Also, by (4), on every step it is multiplied by $\left(1+b_{n}\right)$ and thus it plays the role of an accumulator of $\left\{b_{n}\right\}$. According to the proof of Proposition 4, what $b_{n}$ needs to be 1 or prime is every prime factor of $n$ to appear on $a_{n}$ at least the same number of times, with the single possible exception of one of them.

According to Conjecture 3, we expect a prime $p$ to appear at least $k$ times before getting to $a_{p^{k+1}}$. Since on every step we are multiplying by $\left(1+b_{n}\right)$, in fact primes appear on $a_{n}$ even 


\begin{tabular}{|c|c|c|c|c|}
\hline$n$ & $a_{n-1}$ & $\operatorname{lcm}\left(n, a_{n-1}\right)$ & $b_{n}$ & $a_{n} / a_{n-1}$ \\
\hline 2 & 1 & $2 a_{1}$ & 2 & 3 \\
\hline 3 & 3 & $a_{2}$ & 1 & 2 \\
\hline 4 & $2 \cdot 3$ & $2 a_{3}$ & 2 & 3 \\
\hline 5 & $2 \cdot 3^{2}$ & $5 a_{4}$ & 5 & $2 \cdot 3$ \\
\hline 6 & $2^{2} \cdot 3^{3}$ & $a_{5}$ & 1 & 2 \\
\hline 7 & $2^{3} \cdot 3^{3}$ & $7 a_{6}$ & 7 & $2^{3}$ \\
\hline 8 & $2^{6} \cdot 3^{3}$ & $a_{7}$ & 1 & 2 \\
\hline 9 & $2^{7} \cdot 3^{3}$ & $a_{8}$ & 1 & 2 \\
\hline 10 & $2^{8} \cdot 3^{3}$ & $5 a_{9}$ & 5 & $2 \cdot 3$ \\
\hline 11 & $2^{9} \cdot 3^{4}$ & $11 a_{10}$ & 11 & $2^{2} \cdot 3$ \\
\hline 12 & $2^{11} \cdot 3^{5}$ & $a_{11}$ & 1 & 2 \\
\hline 13 & $2^{12} \cdot 3^{5} \cdot 7$ & $13 a_{12}$ & 13 & $2 \cdot 7$ \\
\hline 14 & $2^{13} \cdot 3^{5} \cdot 7$ & $a_{13}$ & 1 & 2 \\
\hline 15 & $2^{14} \cdot 3^{5} \cdot 7$ & $5 a_{14}$ & 5 & $2 \cdot 3$ \\
\hline 16 & $2^{15} \cdot 3^{6} \cdot 7$ & $a_{15}$ & 1 & 2 \\
\hline$\vdots$ & $\vdots$ & $\vdots$ & $\vdots$ & $\vdots$ \\
\hline
\end{tabular}

Table 4: A deeper explanation of how $a_{n}$ and $b_{n}$ do behave.

\begin{tabular}{|c|c|c|c|}
\hline$n$ & $a_{n-1}$ & $b_{n}$ & $a_{n} / a_{n-1}$ \\
\hline 159 & $2^{212} \cdot 3^{54} \cdot 5^{14} \cdot 7^{14} \cdot 11^{5} \cdot 13 \cdot 17^{3} \cdot 19^{3} \cdot 23 \cdot 31^{2} \cdot 37^{2} \cdot 79$ & 53 & $2 \cdot 3^{3}$ \\
\hline 160 & $2^{213} \cdot 3^{57} \cdot 5^{14} \cdot 7^{14} \cdot 11^{5} \cdot 13 \cdot 17^{3} \cdot 19^{3} \cdot 23 \cdot 31^{2} \cdot 37^{2} \cdot 79$ & 1 & 2 \\
\hline 161 & $2^{214} \cdot 3^{57} \cdot 5^{14} \cdot 7^{14} \cdot 11^{5} \cdot 13 \cdot 17^{3} \cdot 19^{3} \cdot 23 \cdot 31^{2} \cdot 37^{2} \cdot 79$ & 1 & 2 \\
\hline 162 & $2^{215} \cdot 3^{57} \cdot 5^{14} \cdot 7^{14} \cdot 11^{5} \cdot 13 \cdot 17^{3} \cdot 19^{3} \cdot 23 \cdot 31^{2} \cdot 37^{2} \cdot 79$ & 1 & 2 \\
\hline 163 & $2^{216} \cdot 3^{58} \cdot 5^{14} \cdot 7^{14} \cdot 11^{5} \cdot 13 \cdot 17^{3} \cdot 19^{3} \cdot 23 \cdot 31^{2} \cdot 37^{2} \cdot 79$ & 163 & $2^{2} \cdot 41$ \\
\hline 164 & $2^{218} \cdot 3^{58} \cdot 5^{14} \cdot 7^{14} \cdot 11^{5} \cdot 13 \cdot 17^{3} \cdot 19^{3} \cdot 23 \cdot 31^{2} \cdot 37^{2} \cdot 41 \cdot 79$ & 1 & 2 \\
\hline$\vdots$ & $\vdots$ & $\vdots$ & $\vdots$ \\
\hline
\end{tabular}

Table 5: Values of $a_{n-1}$ and $b_{n}$ for $159 \leq n \leq 164$. 


\begin{tabular}{|l||l|l|l|l|l|}
\hline$p \backslash k$ & 2 & 3 & 4 & 5 & 6 \\
\hline \hline 2 & 1 & 3 & 5 & 10 & 17 \\
\hline 3 & 2 & 5 & 11 & 27 & 67 \\
\hline 5 & 1 & 6 & 27 & 110 & 450 \\
\hline 7 & 2 & 11 & 62 & 327 & 1849 \\
\hline 11 & 2 & 21 & 171 & 1487 & 13295 \\
\hline 13 & 1 & 27 & 252 & 2603 & 28150 \\
\hline 17 & 3 & 41 & 502 & 6782 & 94708 \\
\hline 19 & 4 & 52 & 687 & 10128 & 157635 \\
\hline
\end{tabular}

Table 6: First values of $\left\{b_{n}^{s}\right\}$ for $1 \leq s \leq 11$.

more times than expected, specially the smallest. When $n$ grows (Table 5), it seems like we have much more factors than we need. Anyway, a result like the one stated on the Conjecture is needed if we want to prove that there are no composite numbers on the sequence. As we already saw, there is a very strong numeric evidence in order to suspect that it is true. By looking at Table 6, in fact it is clear that the case $k=2$ is the sharpest one. And for it, the number of primes congruent with $p-1$ and less then $p^{2}$ grows with $p$ (see Figure 1).

Acknowledgments: The author thanks Benoit Cloitre for pointing out the reference [Sch] and an anonymous referee for his/her constructive comments.

\section{References}

[CRR11] F. Chamizo, D. Raboso and S. Ruiz-Cabello. On Rowland's sequence. Electron. J. Combin., 18(2): Paper 10, 10, 2011.

[Clo11] B. Cloitre. 10 conjectures in additive number theory. arXiv:1101.4274, 2011.

[Dus02] P. Dussart. Estimates of $\theta(x ; k, l)$ for large values of $x$. Math. Comp. 71 no. 239. 1137-1168, 2002.

[Hea92] D.R. Heath-Brown. Zero-free regions for Dirichlet L-functions, and the least prime in an arithmetic progression. Proc. London Math. Soc. 64(3). 265-338, 1992.

[Lin44] Y.V. Linnik. On the least prime in an arithmetic progression I. The basic theorem. Rec. Math. (Mat. Sbornik) N.S. 15 (57). 139-178, 1944. 
[MV07] H.L. Montgomery, R.C. Vaughan. Multiplicative number theory. I. Classical theory. Cambridge Studies in Advanced Mathematics, 97. Cambridge University Press, 2007.

[Ros41] B. Rosser. Explicit bounds for some functions of prime numbers. Amer. J. Math., 63. 211-232, 1941

[Row08] E. S. Rowland. A natural prime-generating recurrence. J. Integer Seq., 11(2): Article 08.2.8, 13, 2008.

[Sch] M. Schepke. On prime generating sequences. http://www.riemannhypothesis.info/ wp-content/uploads/2014/10/schepke_prime_generating_sequences.pdf

[Xyl11] T. Xylouris. Über die Nullstellen der Dirichletschen L-Funktionen und die Kleinste Primzahl in einer Arithmetischen Progression. PhD Thesis, MathematischNaturwissenschaftliche Fakultät der Universität Bonn, 2011.

Department of Mathematics, Universidad Autónoma de Madrid, 28049 Madrid, Spain

E-mail address: serafin.ruiz@uam.es 